\title{
Strategic approach to control of viral haemorrhagic fever outbreaks in the Eastern Mediterranean Region: report from a regional consultation
}

\author{
M.R. Malik, 'H.E. El Bushra, ${ }^{2}$ M. Opoka, ${ }^{7}$ P. Formenty, ${ }^{3}$ R. Velayudhan ${ }^{4}$ and S. Eremin ${ }^{5}$
}

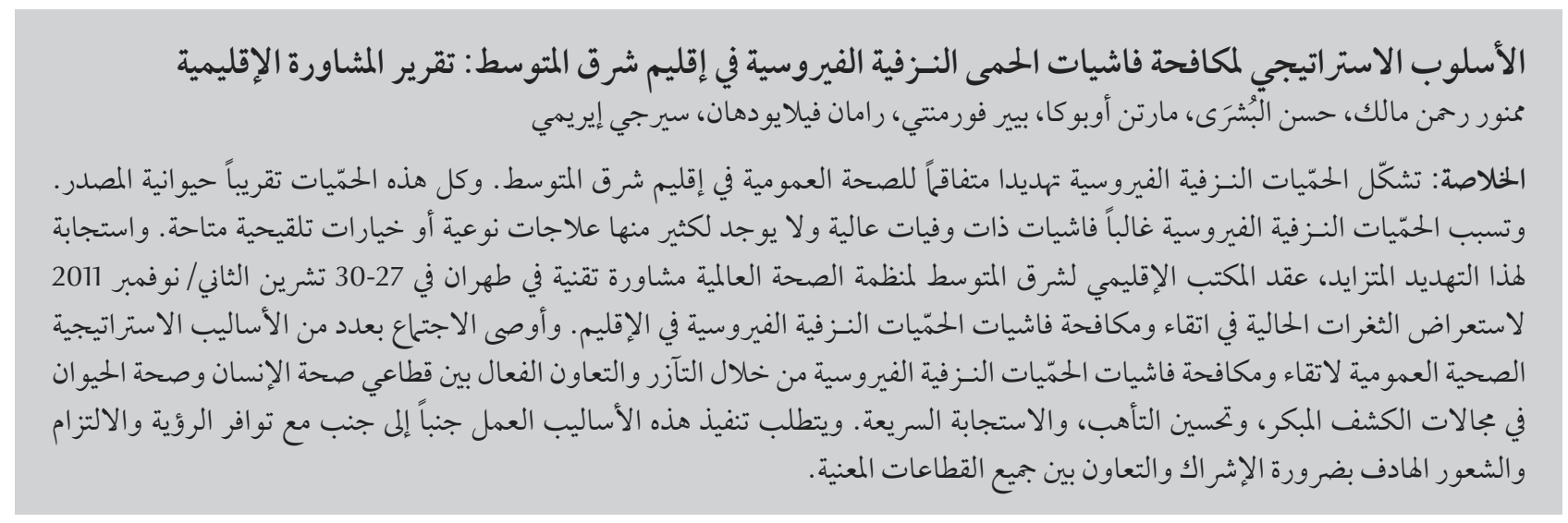

ABSTRACT The viral haemorrhagic fevers (VHF) are a growing public health threat in the Eastern Mediterranean Region. Nearly all of them are of zoonotic origin. VHF often cause outbreaks with high fatalities and, except for yellow fever, currently there are no specific treatment or vaccination options available. In response to this growing threat, the Regional Office for the Eastern Mediterranean of the World Health Organization convened a technical consultation in Tehran on 27-30 November 2011 to review the current gaps in prevention and control of VHF outbreaks in the Region. The meeting recommended a number of strategic public health approaches for prevention and control of VHF outbreaks through synergizing effective collaboration between the human and animal health sectors on areas that involve better preparedness, early detection and rapid response. Implementation of these approaches would require working together with vision, commitment and a sense of purpose involving partnerships and cooperation from all relevant sectors.

Approche stratégique pour lutter contre les flambées de fièvre hémorragique virale dans la Région de la Méditerranée orientale : rapport d'une consultation régionale

RÉSUMÉ Les fièvres hémorragiques virales représentent une menace croissante pour la santé publique dans la Région de la Méditerranée orientale. Elles ont presque toutes une origine zoonotique. Les fièvres hémorragiques virales se propagent souvent sous forme de flambées entraînant une forte mortalité et, à l'exception de la fièvre jaune, il n'existe actuellement pas de traitement spécifique ni de vaccin. En réponse à cette menace croissante, le Bureau régional de l'OMS pour la Méditerranée orientale a convoqué une consultation technique à Téhéran du 27 au 30 novembre 2011 afin d'examiner les lacunes du moment dans la lutte contre les flambées de fièvre hémorragique virale et leur prévention dans la Région. À l'issue de la réunion, les participants ont recommandé un certain nombre d'approches stratégiques en santé publique pour la lutte contre les flambées de fièvre hémorragique virale et leur prévention au moyen d'une collaboration efficace et apportant une synergie entre les secteurs de la santé humaine et de la santé animale dans des domaines nécessitant une détection précoce, une meilleure préparation et une réponse rapide. La mise en ouvre de ces approches appelle un travail conjoint impliquant une vision, un engagement et une détermination associant plusieurs partenariats et coopérations entre les secteurs concernés.

'Pandemic and Epidemic Disease, Division of Communicable Disease Prevention and Control, World Health Organization, Regional Office for Eastern Mediterranean, Cairo, Egypt (Correspondence to M.R. Malik: malikm@who.int). ${ }^{2}$ World Health Organization, Country Office, Beirut, Lebanon. ${ }^{3}$ Emerging and Epidemic Zoonotic Diseases Team, Pandemic and Epidemic Disease Department; ${ }^{4}$ Vector Ecology and Management, Control of Neglected Tropical Disease Department; Infection Control, Pandemic and Epidemic Disease Department, World Health Organization, Geneva, Switzerland. Received: 08/06/12; accepted: 09/12/12 


\section{Introduction}

Viral haemorrhagic fevers (VHF) refer to a group of illnesses that are caused by several distinct families of viruses. While some of the haemorrhagic fever viruses cause relatively mild infection, many of these viruses can cause severe, lifethreatening disease outbreaks with multiple haemorrhagic manifestations [1]. The viruses causing VHF have different modes of transmission and often there is a high risk of health-care-acquired infection. The VHF are a major concern to global health owing to their epidemic potential, high case fatality ratio and the absence of specific treatment and vaccines available to control their spread (with the exception of the yellow fever vaccine) [2].

Human infections with VHF have occurred frequently in the Eastern Mediterranean Region (EMR) in the past, often in epidemic proportions. During the last decade, VHF outbreaks were reported from at least 12 out of the 23 countries in the Region [3]. The Ebola haemorrhagic fever outbreak reported from South Sudan in 2004 was the only VHF outbreak in the region that was caused by a virus (filovirus) belonging to the family Filoviridae [4]. Other haemorrhagic fever outbreaks reported in the Region were caused by arthropod-borne viruses belonging to the family Bunyaviridae and Flaviviridae. These included Alkhurma haemorrhagic fever in Saudi Arabia in 2005 [5], yellow fever in Sudan in 2005 [6], Rift valley fever in Sudan in 2007 [7], dengue/severe dengue (dengue haemorrhagic fever) in Djibouti, Pakistan, Saudi Arabia, Somalia, Sudan and Yemen [8] —almost every year either sporadically or in localized outbreaks - and Crimean-Congo haemorrhagic fever in Afghanistan [9], Islamic Republic of Iran [10], Pakistan [11] and Sudan [12] —in both endemic and epidemic patterns. Apart from causing high numbers of human infections and deaths, these
VHF have frequently led to healthcare-associated transmission among health-care workers in health facilities. In light of the increasing trend, frequency and expansion of geographic distribution of VHF in the Region, the Eastern Mediterranean Regional Office of the World Health Organization (WHO) invited a group of experts to a technical consultation meeting held in Tehran, Islamic Republic of Iran from 27-30 November 2011. The goal of this meeting was to define intervention strategies and public health approaches for prevention and control of VHF outbreaks in the Region. This paper summarizes the strategic public health approach that came out as a result of this consultation as well as the challenges the countries are facing in the Region for their effective control.

\section{Consultation process}

Public health representatives from the countries that are frequently affected by VHF outbreaks in the Region attended this meeting along with a group of WHO experts from the disciplines of entomology, epidemiology, virology and laboratory science, social mobilization and communication, infection control, clinical management and veterinary epidemiology. The experts from WHO had considerable field experience in managing VHF outbreaks in the past. Seven functional domains, representing the overarching public health components for prevention, detection and control of VHF outbreaks were selected and discussed in the meeting (Box 1). In total, 25 participants participated in the discussions and group work of each of the functional domains. Each domain was introduced by a subject matter expert, discussed in the plenary session, and afterwards specific issues related to gaps and constraints in public health control measures were probed in the group work by the participants using scripted templates. At the end of the group work, specific recommendations on public health control measures related to the functional domain were formulated in the plenary through an informed debate which was facilitated by the subject matter expert. The recommendations from each of the functional domains were later synthesized, and final agreement on the intervention strategies was achieved through review of the existing gaps in control measures, the current evidence base, the public health value of each of the recommended strategies and the feasibility of implementation of the recommended strategies in the countries where these diseases are endemic

Seven main strategic public health approaches were thus identified for effective prevention and control of VHF in the EMR.

\section{Improving surveillance for early detection of disease threats in humans}

As most of the VHF are of zoonotic origin, with reservoirs in animals or/ and in arthropods, and the occurrence of such diseases in humans often cannot be precisely predicted, investigation at the first sign of emergence of a new disease in animals is particularly important to detect early any health risk and mitigate its public health impact in humans. As such, the integration of human and animal health surveillance systems is critical through timely gathering and analysis of animal disease data that will enhance early detection of potential infections in humans. The use of information technology will help in designing tailored approaches for sharing these surveillance data in real-time and could reduce the time to detect VHF outbreaks and accelerate control and prevention.

\section{Strengthening laboratory diagnostic capacities for novel pathogens}

Laboratory services would be more effective in early detection of any VHF 


Box 1 Functional domains for discussion at the Eastern Mediterranean Region
technical consultation on viral haemorrhagic fever
\begin{tabular}{|l} 
- Surveillance, early warning and forecasting \\
- Laboratory and diagnostics services \\
- Case management and infection control \\
- Coordination and collaboration \\
- Social mobilization \\
- Epidemic preparedness and response
\end{tabular}

outbreak when there is a common and agreed communication protocol for sharing laboratory surveillance data between animal and human health sectors in real-time. In addition, a mechanism needs to be put in place for sharing of laboratory investigation data within the health sector, principally between the disease surveillance and the clinical services departments. Establishing laboratory networks both within and outside the countries will enhance rapid transfer and shipment of specimens for timely diagnosis of VHF.

\section{Improving case management and infection control}

To ensure preparedness of health care facilities to VHF outbreaks, an infection prevention and control program should be implemented before an outbreak strikes, with the standard precautions as an essential component. Standard precautions should be used in the care and treatment of all patients irrespective of their perceived or confirmed infectious status. If consistently applied, the standard precautions would help prevent most transmission through exposure to blood and body fluids before VHF is recognized. Development and implementation of a standard protocol for clinical case management of VHF will allow management of cases appropriately, maintaining additional infection control precautions including isolation of patients when needed. Standardization of the treatment protocol should be backed up with training of health-care workers on case management and infection control measures as well pre-positioning of strategic supplies. As the clinical manifestations of some arboviral diseases often clinically resemble each other, thus leading to confusion and misdiagnosis by health-care workers, the use of a clinical decision algorithm for acute febrile illnesses with a more sensitive case definition will be useful for early detection of any suspected cases. Use of such a decision tree will help in guiding initial therapeutic decisions and trigger the protocol steps for further laboratory diagnosis and follow-up.

\section{Building effective collaboration between animal and human health sectors}

As it is difficult to predict when or where the next VHF outbreak will emerge, close collaboration between veterinary and public health specialists is important. The goal of this intersectoral collaboration would be to enhance inter-personal and interorganizational communication. An inter-agency taskforce can lead this process of fostering collaboration through regular exchange of scientific information proactively between the 2 sectors. The task force can also guide joint field investigation and share institutional resources within a coordinated framework of partnerships and agreement for the One Health concept. This would synergize effective prevention and control efforts at the animal-human interface.

\section{Integrating vector control management}

An integrated vector control management (IVM) approach should be considered for all arthropod-borne viruses as the most rational decisionmaking process to optimize the use of resources for effective vector control. The IVM strategy is based on evidence and integrated management of mosquito vectors including rodents, and promotes the use of a range of interventions, either alone or in combination, which are selected on the basis of local knowledge about the vectors, diseases and disease determinants. IVM would therefore be the most effective strategy for the control of vectors including rodents that are responsible for transmission of a number of arthropod-borne viral haemorrhagic fevers. As a first step, countries need to carry out a detailed mapping of the vectors and their breeding sites to identify the spatio-temporal distribution of the vector species. Such information may be useful for targeting control measures for breeding sites during the inter-epidemic period. The vector control measures should seek to reduce the potential breeding sites of adult mosquito populations or their interactions with human below that which can sustain an epidemic. Setting up a sentinel site for entomological surveillance in areas of high vector densities and integrating it with that of epidemiological and viral surveillance systems can provide meaningful information through reporting of unusual clusters of acute febrile illnesses, a suddenly rise in vector density or a fortuitous isolation of a novel arbovirus. Such information can be helpful to understand the anticipated, prevailing or evolving risk. 


\section{Reducing transmission through social and behavioural interventions}

The success or failure of interrupting the transmission chain for most of the VHF, especially those involving intermediate vertebrate hosts, will rely on the relevance of the behavioural response of the exposed populations. To design appropriate social and behavioural interventions for VHF, the community's risk perception and how this relate to actual or intended behaviour, socio- or psycho-cognitive factors that characterize the exposed population's behaviour and the cultural factors that influence protective factors and sustainability of adherence to protective behaviour will need to be considered.

\section{Developing epidemic preparedness and response capacities for VHF}

The main strategy should begin with developing a national plan involving all important stakeholders. The plan should consider developing a geographic map of the distribution of VHF occurring in the countries using geographic information systems and other information technologies, and conducting a detailed risk assessment. Furthermore, areas at risk for expansion of VHF should also be identified. Human, animal and vector surveillance should be strengthened and if possible integrated with data and shared, so that vital information on risks are exchanged on a regular basis between the partners through a well-coordinated mechanism. The plan should encompass pre-positioning of drugs and other strategic supplies (investigation kits, personal protective equipment, etc.), development of appropriate guidelines and standards to measure the effectiveness of response operations and public education programmes aimed at limiting exposure to risk. Setting up a multidisciplinary coordinating body to foster collaboration and integration between all partners and to guide, lead and provide emergency response operations during an outbreak would be the key for a successful epidemic preparedness and response plan.

\section{Discussion}

In recent times, emerging infectious diseases including the VHF have become of increasing concern. The occurrence and spread of VHF has been facilitated by the increased volume of international travel, including transboundary mass population and livestock movements within neighbouring countries [13]. As the world is becoming increasingly interconnected, VHF in one country can potentially constitute a threat to global health security. Globalization has resulted in the unparalleled passage of people, animals and goods across national borders, which in turn has fuelled the international spread of infectious diseases. Many of the VHF are transboundary zoonotic diseases; they spread across borders from their origin to impact on trade, commerce, tourism and consumer confidence with devastating economic consequences. Therefore, regional cooperation and cross-border collaboration in terms of timely sharing and exchange of key information on animal and human diseases such as the VHF will enhance better prevention and control of these diseases.

The experiences drawn from EMR countries have shown that most of the VHF outbreaks have occurred in remote areas, making it sometimes impossible to provide public health services to these hard-to-reach populations. The detection and diagnosis of the disease has been considerably delayed due to difficulties in deploying teams for field investigation, lack of appropriate and safe sample shipment mechanism, lack of appropriate laboratory diagnostic facilities on-site or in-country, and insufficient capacities of the countries to plan, mobilize and implement appropriate control measures in such settings and to monitor the progress of control measures in geographically dispersed areas. The detection of these disease threats require functional sub-national surveillance capacity and therefore the need for investing in strengthening subnational outbreak surveillance and response capacities in the countries frequently affected by these diseases.

Most novel viral pathogens causing haemorrhagic fevers that have emerged or re-emerged worldwide in humans originated from animals (specifically wildlife) or from products of animal origin. Knowledge of extra-human reservoirs of these pathogens remains essential for understanding the epidemiology and potential control measures of these zoonotic diseases.

The EMR meeting outlined above recognized that there is inadequate transparency regarding timely reporting of VHF to WHO or any other international agency that is mandated to investigate and respond appropriately for global health security. Often medical authorities in the countries deny the existence of human cases, making it difficult for these agencies to understand the epidemiology, disease progression and use the opportunity to understand which methods work and do not work for control of these diseases in different settings.

In the light of some of the challenges identified in the meeting, the participants acknowledged that another strategic approach that merits attention is strengthening cross-border collaboration, such as the mechanisms established between the Islamic Republic of Iran, Iraq, Pakistan, Afghanistan and WHO for cross-border surveillance, and creating a roster of experts in different fields and areas of expertise. As the meeting recognized that the affected countries in the Region vary significantly in terms of their public health capacity to respond to outbreaks and also to prevent and control these infectious diseases, a comprehensive inventory of Regional resources and development of networks for surveillance, infection 
control and laboratory diagnostic at national and regional levels will greatly improve Regional capabilities to respond to any VHF disease outbreak in the EMR with mobilization of appropriate and skilful medical, public health and scientific resources and expertise. Such mechanisms will enhance the public health preparedness and readiness of the Region.

\section{Conclusion}

A number of challenges were identified that exist in many countries in the EMR for prevention and control of VHF outbreaks (Box 2). Given the scale and magnitude of these threats, a strategy to protect public health against VHF outbreaks through appropriate prevention, preparedness, early detection and control measures were considered of paramount importance. The strategy should encompass improvement in surveillance, laboratory diagnosis, integrated vector control, social and behavioural interventions, clinical and infection control practices for the VHF case management, as well as promoting cross-border collaboration between neighbouring countries and strengthening inter-agency and cross-sectoral collaboration between the animal and human health sectors. The prevention, control and mitigation of public health threats from emergent infectious diseases such as those of the VHF remain a critical challenge for health security in the region. The way forward will require collaboration from all partners with strategic vision, commitment and a sense of purpose to address these perpetual health risks in the EMR. International organizations, including WHO, need to take a leading role in coordinating and streamlining technical support that may be required for implementation of these strategic directions to ensure health security in the Region.

Competing interests: None declared.
Box 2 Challenges for prevention and control of viral haemorrhagic fevers (VHF) in the Eastern Mediterranean Region

\section{Organizational challenges}

- Poor level of awareness among policy and decision-makers about the serious nature of the disease.

- Presence of other competing health priorities that often take precedence.

- Being zoonotic in nature, control of VHF is mostly dependent on its control in animal reservoirs.

- Lack of transparency of countries in reporting outbreaks, and countries' denial of the existence of human cases.

- Poor level of inter-sectoral collaboration and cooperation between public health and veterinary health authorities.

- Lack of integration between human and veterinary medicine for exchange of epidemiological and laboratory surveillance data of the animal and human health sectors.

- Absence of cross-talk within the health sector between the surveillance, clinical services and laboratory services departments.

\section{Diagnosis and detection}

- Occurrence of VHF in remote areas makes it impossible to provide services rapidly to hard-to-reach populations.

- Lack of laboratory diagnostic capabilities in countries to detect novel viral pathogens.

- Difficulties in international transfer of samples for logistic and economic reasons.

- Difficulties in conducting field investigation in remote areas where most of the VHF occur.

- Lack of clear understanding of the epidemiology and disease manifestations of VHF.

- Problems in differential diagnosis due to the clinical symptoms of many of VHF resembling each other and often overlapping.

\section{Control and interruption of transmission}

- Insufficient capacities of countries to plan, mobilize and implement appropriate control measures.

- High probability of nosocomial transmission of some of the filo- and arboviral haemorrhagic fevers in health-care settings.

- Poor application of strict barrier nursing and other appropriate infection control measures in health-care facilities.

- Lack of information on high-risk behaviours, including cultural and social factors, that are associated with risk of transmission of VHF.

- Inappropriate or inadequate vector control operations.

- Lack or insufficient evidence on some of the public health control measures. 


\section{References}

1. Viral haemorrhagic fevers. Fact sheet. US Centers for Disease Control and Prevention (http://www.cdc.gov/ncidod/dvrd/ spb/mnpages/dispages/vhf.htm, accessed 4 August 2013).

2. Gould EA, Solomon T. Pathogenic flaviviruses. Lancet, 2008, 371:500-509.

3. Growing threat of viral haemorrhagic fever in the Eastern Mediterranean Region: a call for action. Technical paper. 54th session of the Regional Committee for the Eastern Mediterranean. Agenda item 6(c). Cairo, World Health Organization Regional Office for the Eastern Mediterranean, 2007 (EM/RC54/5).

4. Outbreak of Ebola haemorrhagic fever in Yambio, south Sudan, April-June 2004. Weekly Epidemiological Record, 2005, 80:370-375.

5. Madani TA. Alkhumra virus infection, a new viral hemorrhagic fever in Saudi Arabia. Journal of Infection, 2005, 51:91-97.

6. Gould LH et al. An outbreak of yellow fever with concurrent chikungunya virus transmission in South Kordofan, Sudan, 2005. Transactions of the Royal Society of Tropical Medicine and Hygiene, 2008, 102:1247-1254.

7. Hassan OA et al. The 2007 Rift Valley fever outbreak in Sudan. PLoS Neglected Tropical Diseases, 2011, 5:e1229.
8. Dengue: call for urgent intervention for a rapidly expanding emerging disease. Technical paper. 58th session of the Regional Committee for the Eastern Mediterranean. Agenda item 4(a). Cairo, World Health Organization Regional Office for the Eastern Mediterranean, 2011 (EM/RC58/3).

9. Mofleh J, Ahmad AZ. Crimean-Congo haemorrhagic fever outbreak investigation in the Western Region of Afghanistan in 2008. Eastern Mediterranean Health Journal, 2012, 18:522-526.

10. Mardani $\mathrm{M}$ et al. Crimean-Congo hemorrhagic fever among health care workers in Iran: a seroprevalence study in two endemic regions. American Journal of Tropical Medicine and Hygiene, 2007, 76:443-445.

11. Sheikh AS et al. Bi-annual surge of Crimean-Congo haemorrhagic fever (CCHF): a five-year experience. International Journal of Infectious Diseases, 2005, 9:37-42.

12. Elata AT et al. A nosocomial transmission of Crimean-Congo hemorrhagic fever to an attending physician in north Kordufan, Sudan. Virology Journal, 2011, 8:303.

13. Richt JA, Feldmann H. Emerging zoonoses: recent advances and future challenges. Zoonoses and Public Health, 2009, $56: 257$ 